\title{
Preis-Special für Späteinsteiger
}

Sie sind begeisterter Testhörer der Akademie-Online-Kurse und haben bisher den kompletten Jahreskurs nur noch nicht gebucht, weil Sie denken: im Sommer lohnt sich's nicht mehr? Dann kommt jetzt das richtige Einsteiger-Angebot für Sie: Ab dem 01.07.2016 buchen Sie das Akademie-Online-Jahresabo zum halben Preis!

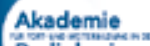

AKADEMIE

FÖR FORT- UND WEITERBILDUNG IN DER RADIOLOGIE

in der Deutschen Rontgengesellschaft e. $\mathrm{V}$.

Vom 01.07. bis 31.12.2016 warten insgesamt noch zwölf ärztliche Online-Kurse und sechs MTRA-Online-Kurse zu unschlagbar günstigen Konditionen auf

\begin{tabular}{l}
\hline Tarifgruppe \\
\hline Fachärztin / Facharzt - Mitglied DRG / BDR / \\
ÖRG oder SRG-SSR \\
\hline Fachärztin / Facharzt - ohne Mitgliedschaft \\
\hline AiW - Mitglied DRG / BDR / ÖRG oder SRG-SSR \\
\hline AiW - ohne Mitgliedschaft \\
\hline Ärztin / Arzt in Erziehungsurlaub \\
\hline MTRA - VMTB-Mitglied \\
\hline MTRA - ohne Mitgliedschaft \\
\hline
\end{tabular}

Sie. Und das Beste daran ist: Als Halbjahresabonnent haben Sie Zugriff auf alle Kursaufzeichnungen ab dem 01.07.2016 und können verpasste Kurse noch ein- mal bequem für 14 bzw. 28 Tage nachträglich ansehen.

Für jedes besuchte Live-Webinar erhalten Sie eine Teilnahmebescheinigung. Alle Kurse sind mit zwei CME-Punkten der Akademie zertifiziert; alle ärztlichen Kurse sind zusätzlich mit jeweils zwei Fortbildungspunkten der Ärztekammer Hessen zertifiziert.

Eine Übersicht über die neuen Aktionspreise finden Sie in der unten stehenden Tabelle; die Tarife sind ab dem 1. Juli freigeschaltet. Hier können Sie sich anmelden: drgakademie.de > Akademie Online $>$ Anmeldung

\section{Aktionspreis Halbjahresabo}

Normales Jahresabo
(Teilnahme und Zugang zum Streambereich für alle Ärzte- und MTRA-Kurse vom 01.01.2016 31.12.2016)

$200 €$

(Teilnahme und Zugang zum Streambereich für alle Ärzte- und MTRA-Kurse vom 01.07.2016 31.12.2016)

$100 €$

$400 €$

$200 €$

$80 €$

$40 €$

$160 €$

$80 €$

$80 €$

$40 €$

$60 €$

$30 €$

$140 €$

$70 €$ 